\title{
Generalization of two identities in Ramanujan's lost notebook
}

\author{
by \\ Youn-Seo Choi (Seoul)
}

1. Introduction. In his last letter to G. H. Hardy [3], S. Ramanujan described a mock theta function to be a function $f(q)$ defined by a $q$-series which converges for $|q|<1$ and which satisfies the following two conditions:

(0) For every root of unity $\zeta$, there is a theta function $\theta_{\zeta}(q)$ such that the difference $f(q)-\theta_{\zeta}(q)$ is bounded as $q \rightarrow \zeta$ radially.

(1) There is no single theta function which works for all $\zeta$ : i.e., for every theta function $\theta(q)$ there is some root of unity $\zeta$ for which $f(q)-\theta(q)$ is unbounded as $q \rightarrow \zeta$ radially.

He then provided a long list of "third order", "fifth order", and "seventh order" mock theta functions together with identities satisfied by them. G. N. Watson $[11,12]$ proved these mock theta function identities, and introduced three new third order mock theta functions. Further identities can be found in Ramanujan's lost notebook [10]. Among them, G. E. Andrews and F. G. Garvan [1] examined ten identities for Ramanujan's fifth order mock theta functions, and showed that the first five identities are equivalent to each other, and the other five identities are also equivalent. They called these "the mock theta conjectures". Later, D. Hickerson [8] proved these identities, and then [9] he provided two new results for the seventh order mock theta functions which do not appear in Ramanujan's letter. Recently, the author $[5,6]$ proved certain tenth order mock theta function identities from the lost notebook. There are two additional results related to Ramanujan's mock theta functions in the lost notebook.

In his lost notebook [10, p. 18], Ramanujan defined two functions

$$
\Psi(q):=\sum_{n=0}^{\infty} \frac{q^{5 n^{2}}}{\left(q^{2} ; q^{5}\right)_{n+1}\left(q^{3} ; q^{5}\right)_{n}}, \quad \Phi(q):=\sum_{n=0}^{\infty} \frac{q^{5 n^{2}}}{\left(q ; q^{5}\right)_{n+1}\left(q^{4} ; q^{5}\right)_{n}},
$$

2000 Mathematics Subject Classification: Primary 11B65; Secondary 33C20.

This work was supported by Korea Research Foundation Grant (KRF-2003-003C00002). 
where $(a ; q)_{n}:=\prod_{m=0}^{\infty}\left(1-a q^{m}\right) / \prod_{m=0}^{\infty}\left(1-a q^{n+m}\right)$ for an integer $n$ and complex numbers $a, q$ with $|q|<1$. These functions also appear in the mock theta conjectures

$$
\begin{aligned}
& f_{0}(q)=\frac{\left(q^{5} ; q^{5}\right)_{\infty}\left(q^{5} ; q^{10}\right)_{\infty}}{\left(q ; q^{5}\right)_{\infty}\left(q^{4} ; q^{5}\right)_{\infty}}+2-2 \Phi\left(q^{2}\right), \\
& f_{1}(q)=\frac{\left(q^{5} ; q^{5}\right)_{\infty}\left(q^{5} ; q^{10}\right)_{\infty}}{\left(q^{2} ; q^{5}\right)_{\infty}\left(q^{3} ; q^{5}\right)_{\infty}}+\frac{2}{q}-\frac{2}{q} \Psi\left(q^{2}\right),
\end{aligned}
$$

where $(a ; q)_{\infty}:=\prod_{m=0}^{\infty}\left(1-a q^{m}\right)$, and

$$
f_{0}(q):=\sum_{n=0}^{\infty} \frac{q^{n^{2}}}{(-q ; q)_{n}}, \quad f_{1}(q):=\sum_{n=0}^{\infty} \frac{q^{n^{2}+n}}{(-q ; q)_{n}}
$$

are fifth order mock theta functions. He also provided the following two identities involving $\Psi(q)$ and $\Phi(q)$ which have not heretofore been proved:

$$
\begin{aligned}
& \frac{1}{q}\left(\Psi\left(q^{3}\right)-1\right)+\frac{\left(q^{5} ; q^{5}\right)_{\infty}^{2}}{\left(q^{15} ; q^{15}\right)_{\infty}\left(q ; q^{5}\right)_{\infty}\left(q^{4} ; q^{5}\right)_{\infty}} \\
& \quad=\sum_{n=0}^{\infty} \frac{q^{15 n^{2}}}{\left(q ; q^{15}\right)_{n+1}\left(q^{14} ; q^{15}\right)_{n}}-1+\sum_{n=0}^{\infty} \frac{q^{15 n^{2}}}{\left(q^{4} ; q^{15}\right)_{n+1}\left(q^{11} ; q^{15}\right)_{n}}
\end{aligned}
$$

and

$$
\begin{gathered}
\Phi\left(q^{3}\right)-1+\sum_{n=0}^{\infty} \frac{q^{15 n^{2}}}{\left(q^{2} ; q^{15}\right)_{n+1}\left(q^{13} ; q^{15}\right)_{n}} \\
-\frac{1}{q^{2}}\left(\sum_{n=0}^{\infty} \frac{q^{15 n^{2}}}{\left(q^{7} ; q^{15}\right)_{n+1}\left(q^{8} ; q^{15}\right)_{n}}-1\right) \\
=\frac{\left(q^{5} ; q^{5}\right)_{\infty}^{2}}{\left(q^{15} ; q^{15}\right)_{\infty}\left(q^{2} ; q^{5}\right)_{\infty}\left(q^{3} ; q^{5}\right)_{\infty}} .
\end{gathered}
$$

We will prove (1) and (2). We will moreover give seven identities which are analogues of (1) and (2), and are also related to Ramanujan's mock theta functions.

Define

$$
\psi(q):=\sum_{n=1}^{\infty} \frac{q^{n^{2}}}{\left(q ; q^{2}\right)_{n}}, \quad \omega(q):=\sum_{n=0}^{\infty} \frac{q^{2 n(n+1)}}{\left(q ; q^{2}\right)_{n+1}^{2}} .
$$

$\psi(q)$ is a third order mock theta function which appears in Ramanujan's letter, and $\omega(q)$ is also a third order mock theta function which does not appear in Ramanujan's letter, but was introduced by Watson [11]. We will prove two identities involving $\psi(q)$ and $\omega(q)$, respectively, which are not 
established by Ramanujan:

$$
\begin{array}{r}
\psi\left(q^{3}\right)+\sum_{n=0}^{\infty} \frac{q^{12 n^{2}}}{\left(q ; q^{12}\right)_{n+1}\left(q^{11} ; q^{12}\right)_{n}}-\frac{1}{q^{3}}\left(\sum_{n=0}^{\infty} \frac{q^{12 n^{2}}}{\left(q^{7} ; q^{12}\right)_{n+1}\left(q^{5} ; q^{12}\right)_{n}}-1\right) \\
=\frac{\left(q^{4} ; q^{4}\right)_{\infty}^{2}}{\left(q^{12} ; q^{12}\right)_{\infty}\left(q ; q^{4}\right)_{\infty}\left(q^{3} ; q^{4}\right)_{\infty}}
\end{array}
$$

and

$$
q^{2} \omega\left(q^{3}\right)+\frac{\left(q^{2} ; q^{2}\right)_{\infty}^{2}}{\left(q^{6} ; q^{6}\right)_{\infty}\left(q ; q^{2}\right)_{\infty}^{2}}=2 \sum_{n=0}^{\infty} \frac{q^{6 n^{2}}}{\left(q ; q^{6}\right)_{n+1}\left(q^{5} ; q^{6}\right)_{n}}-1
$$

Define

$$
\begin{gathered}
\psi_{0}(q):=\sum_{n=0}^{\infty} \frac{q^{7 n^{2}}}{\left(q ; q^{7}\right)_{n+1}\left(q^{6} ; q^{7}\right)_{n}}, \quad \psi_{1}(q):=\sum_{n=0}^{\infty} \frac{q^{7 n^{2}}}{\left(q^{2} ; q^{7}\right)_{n+1}\left(q^{5} ; q^{7}\right)_{n}}, \\
\psi_{2}(q):=\sum_{n=0}^{\infty} \frac{q^{7 n^{2}}}{\left(q^{3} ; q^{7}\right)_{n+1}\left(q^{4} ; q^{7}\right)_{n}} .
\end{gathered}
$$

Hickerson [9] proved three of Ramanujan's seventh order mock theta function identities which represent three seventh order mock theta functions by theta functions and Lambert series. These identities are equivalent to the following identities which involve $\psi_{0}(q), \psi_{1}(q)$, and $\psi_{2}(q)$ :

$$
\begin{gathered}
F_{0}(q)=2 \psi_{0}(q)-\frac{f\left(-q^{3},-q^{4}\right)^{2}}{(q ; q)_{\infty}}, \quad F_{1}(q)=2 \psi_{1}(q)+\frac{q f\left(-q,-q^{6}\right)^{2}}{(q ; q)_{\infty}}, \\
F_{2}(q)=2 q^{-1}\left(\psi_{2}(q)-1\right)+\frac{f\left(-q^{2},-q^{5}\right)^{2}}{(q ; q)_{\infty}}
\end{gathered}
$$

where

$$
F_{0}(q):=\sum_{n=0}^{\infty} \frac{q^{n^{2}}}{\left(q^{n+1} ; q\right)_{n}}, \quad F_{1}(q):=\sum_{n=1}^{\infty} \frac{q^{n^{2}}}{\left(q^{n} ; q\right)_{n}}, \quad F_{2}(q):=\sum_{n=0}^{\infty} \frac{q^{n^{2}+n}}{\left(q^{n+1} ; q\right)_{n+1}}
$$

are the seventh order mock theta functions which were defined by Ramanujan in his letter. We will prove three new identities involving $\psi_{0}(q), \psi_{1}(q)$, and $\psi_{2}(q)$, respectively, which are not established by Ramanujan:

$$
\begin{gathered}
\psi_{0}\left(q^{3}\right)-1+\sum_{n=0}^{\infty} \frac{q^{21 n^{2}}}{\left(q^{4} ; q^{21}\right)_{n+1}\left(q^{17} ; q^{21}\right)_{n}} \\
-\frac{1}{q^{3}}\left(\sum_{n=0}^{\infty} \frac{q^{21 n^{2}}}{\left(q^{10} ; q^{21}\right)_{n+1}\left(q^{11} ; q^{21}\right)_{n}}-1\right) \\
=\frac{\left(q^{7} ; q^{7}\right)_{\infty}^{2}}{\left(q^{21} ; q^{21}\right)_{\infty}\left(q^{3} ; q^{7}\right)_{\infty}\left(q^{4} ; q^{7}\right)_{\infty}}
\end{gathered}
$$


(6)

$$
\begin{gathered}
\psi_{1}\left(q^{3}\right)-1+\sum_{n=0}^{\infty} \frac{q^{21 n^{2}}}{\left(q ; q^{21}\right)_{n+1}\left(q^{20} ; q^{21}\right)_{n}} \\
-\frac{1}{q}\left(\sum_{n=0}^{\infty} \frac{q^{21 n^{2}}}{\left(q^{8} ; q^{21}\right)_{n+1}\left(q^{13} ; q^{21}\right)_{n}}-1\right) \\
=\frac{\left(q^{7} ; q^{7}\right)_{\infty}^{2}}{\left(q^{21} ; q^{21}\right)_{\infty}\left(q ; q^{7}\right)_{\infty}\left(q^{6} ; q^{7}\right)_{\infty}}
\end{gathered}
$$

(7) $\quad \psi_{2}\left(q^{3}\right)-1+\frac{\left(q^{7} ; q^{7}\right)_{\infty}^{2}}{\left(q^{21} ; q^{21}\right)_{\infty}\left(q^{2} ; q^{7}\right)_{\infty}\left(q^{5} ; q^{7}\right)_{\infty}}$

$$
=\sum_{n=0}^{\infty} \frac{q^{21 n^{2}}}{\left(q^{2} ; q^{21}\right)_{n+1}\left(q^{19} ; q^{21}\right)_{n}}-1+\sum_{n=0}^{\infty} \frac{q^{21 n^{2}}}{\left(q^{5} ; q^{21}\right)_{n+1}\left(q^{16} ; q^{21}\right)_{n}} .
$$

Define

$$
\phi_{0}(q):=\sum_{n=0}^{\infty} \frac{(-1)^{n}\left(q^{5} ; q^{10}\right)_{n} q^{5 n^{2}}}{\left(q^{2} ; q^{10}\right)_{n+1}\left(q^{8} ; q^{10}\right)_{n}}, \quad \phi_{1}(q):=\sum_{n=0}^{\infty} \frac{(-1)^{n}\left(q^{5} ; q^{10}\right)_{n} q^{5 n^{2}}}{\left(q^{4} ; q^{10}\right)_{n+1}\left(q^{6} ; q^{10}\right)_{n}} .
$$

The author [4] established two of Ramanujan's tenth order mock theta function identities which represent tenth order mock theta functions by theta functions and Lambert series. These identities are equivalent to the following identities which involve $\phi_{0}(q)$ and $\phi_{1}(q)$ :

$$
\begin{aligned}
& X(q)=-\frac{\left(q^{5} ; q^{5}\right)_{\infty}\left(q^{10} ; q^{10}\right)_{\infty} f\left(-q^{2},-q^{3}\right)}{f\left(-q^{2},-q^{8}\right) f\left(-q,-q^{4}\right)}+2 \phi_{0}(q), \\
& \chi(q)=2+q \frac{\left(q^{5} ; q^{5}\right)_{\infty}\left(q^{10} ; q^{10}\right)_{\infty} f\left(-q,-q^{4}\right)}{f\left(-q^{4},-q^{6}\right) f\left(-q^{2},-q^{3}\right)}-2 \phi_{1}(q),
\end{aligned}
$$

where

$$
X(q):=\sum_{n=0}^{\infty} \frac{(-1)^{n} q^{n^{2}}}{(-q ; q)_{2 n}}, \quad \chi(q):=\sum_{n=0}^{\infty} \frac{(-1)^{n} q^{(n+1)^{2}}}{(-q ; q)_{2 n+1}} .
$$

These functions $X(q)$ and $\chi(q)$ are the tenth order mock theta functions which were defined by Ramanujan in his lost notebook. We will prove two new identities involving $\phi_{0}(q)$ and $\phi_{1}(q)$, respectively, which were not established by Ramanujan:

$$
\begin{array}{r}
\phi_{0}\left(q^{2}\right)-\frac{1}{q^{4}}\left(\sum_{n=0}^{\infty} \frac{(-1)^{n}\left(q^{10} ; q^{20}\right)_{n} q^{10 n^{2}}}{\left(q^{14} ; q^{20}\right)_{n+1}\left(q^{6} ; q^{20}\right)_{n}}-1\right) \\
=\frac{\left(q^{10} ; q^{10}\right)_{\infty}^{2}}{\left(q^{4} ; q^{10}\right)_{\infty}\left(q^{6} ; q^{10}\right)_{\infty} f\left(q^{10}, q^{30}\right)}
\end{array}
$$




$$
\begin{aligned}
\phi_{1}\left(q^{2}\right)-\frac{1}{q^{8}}\left(\sum_{n=0}^{\infty} \frac{(-1)^{n}\left(q^{10} ; q^{20}\right)_{n} q^{10 n^{2}}}{\left(q^{18} ; q^{20}\right)_{n+1}\left(q^{2} ; q^{20}\right)_{n}}-1\right) \\
=\frac{\left(q^{10} ; q^{10}\right)_{\infty}^{2}}{\left(q^{2} ; q^{10}\right)_{\infty}\left(q^{8} ; q^{10}\right)_{\infty} f\left(q^{10}, q^{30}\right)} .
\end{aligned}
$$

In this paper, we obtain two theorems, which contain identities (1)-(9) as special cases.

TheOREm 1. For a complex number $q$ with $|q|<1$, and $x$ neither 0 nor an integral power of $q$,

$$
\begin{aligned}
& \frac{(q ; q)_{\infty}^{2}}{\left(q^{3} ; q^{3}\right)_{\infty}(x ; q)_{\infty}\left(x^{-1} q ; q\right)_{\infty}}=\sum_{n=0}^{\infty} \frac{q^{3 n^{2}}}{\left(x ; q^{3}\right)_{n+1}\left(x^{-1} q^{3} ; q^{3}\right)_{n}}-1 \\
& -x^{-1}\left(\sum_{n=0}^{\infty} \frac{q^{3 n^{2}}}{\left(x q ; q^{3}\right)_{n+1}\left(x^{-1} q^{2} ; q^{3}\right)_{n}}-1\right)+\sum_{n=0}^{\infty} \frac{q^{3 n^{2}}}{\left(x^{-1} q ; q^{3}\right)_{n+1}\left(x q^{2} ; q^{3}\right)_{n}}
\end{aligned}
$$

TheOREM 2. For a complex number $q$ with $|q|<1$, and $x$ neither 0 nor an integral power of $q$,

$$
\begin{aligned}
\frac{\left(q^{2} ; q^{2}\right)_{\infty}^{2}}{f\left(q^{2}, q^{6}\right)\left(x ; q^{2}\right)_{\infty}\left(x^{-1} q^{2} ; q^{2}\right)_{\infty}}= & \sum_{n=0}^{\infty} \frac{(-1)^{n}\left(q^{2} ; q^{4}\right)_{n} q^{2 n^{2}}}{\left(x ; q^{4}\right)_{n+1}\left(x^{-1} q^{4} ; q^{4}\right)_{n}} \\
& -x^{-1}\left(\sum_{n=0}^{\infty} \frac{(-1)^{n}\left(q^{2} ; q^{4}\right)_{n} q^{2 n^{2}}}{\left(x q^{2} ; q^{4}\right)_{n+1}\left(x^{-1} q^{2} ; q^{4}\right)_{n}}-1\right) .
\end{aligned}
$$

The main purpose of this paper is to prove Theorem 1, Theorem 2, two identities in Ramanujan's lost notebook, and the seven new identities above.

In Section 2, we first prove Theorems 1 and 2 using the preliminary results and transformation formulas for $g(x, q), h(x, q)$, and the hypergeometric series. In Section 3, using Theorem 1, we prove the identities (1) and (2) from Ramanujan's lost notebook. In Section 4, we prove the identities (3) and (4) by using Theorem 1 . In Section 5 , we discuss the relation between Ramanujan's seventh order mock theta functions and the functions $\psi_{0}(q)$, $\psi_{1}(q)$, and $\psi_{2}(q)$, and prove the identities (5)-(7) by using Theorem 1 . In Section 6, we discuss the relation between Ramanujan's tenth order mock theta functions and the two functions $\phi_{0}(q)$ and $\phi_{1}(q)$, and prove the two identities (8) and (9) by using Theorem 2.

2. Proofs of Theorems 1 and 2. In this section, we prove the main theorems of this paper. We need the following definitions, theorems, and lemmas. 
Definition (Ramanujan's general theta function). For complex numbers $q, b$, and $c$ with $|q|<1$ and $|b c|<1$, and any integer $n$,

$$
f(b, c):=\sum_{j=-\infty}^{\infty} b^{j(j+1) / 2} c^{j(j-1) / 2}=(-b ; b c)_{\infty}(-c ; b c)_{\infty}(b c ; b c)_{\infty}
$$

Recall that the second equation above is called the Jacobi triple product identity.

Definition. Let $q$ be any complex number with $|q|<1$, and let $x$ be neither 0 nor an integral power of $q$. Define

$$
\begin{aligned}
g(x, q) & :=x^{-1}\left(\sum_{n=0}^{\infty} \frac{q^{n^{2}}}{(x ; q)_{n+1}\left(x^{-1} q ; q\right)_{n}}-1\right), \\
h(x, q) & :=\sum_{n=0}^{\infty} \frac{(-1)^{n}\left(q^{1 / 2} ; q\right)_{n} q^{n^{2} / 2}}{(x ; q)_{n+1}\left(x^{-1} q ; q\right)_{n}} .
\end{aligned}
$$

Hickerson discussed $g(x, q)$ in his paper [9]. Next, we give the transformation formula for $g(x, q)$.

TheOREm 3 ([8, Theorem 2.0, p. 649]). For a complex number $q$ with $|q|<1$, and $x$ neither 0 nor an integral power of $q$,

$$
(q ; q)_{\infty} g(x, q)=\sum_{n=-\infty}^{\infty} \frac{(-1)^{n} q^{3 n(n+1) / 2}}{1-x q^{n}}
$$

To derive the transformation formula for $h(x, q)$, we need to use the following theorem.

Theorem 4 (Watson's transformation formula, [11, p. 63]). If $q, a, b$, and $c$ with $|q|<1$ are complex numbers, and $d$, $e$, or $f$ is of the form $q^{-n}$, where $n$ is a positive integer, then

$$
\begin{aligned}
\sum_{n=0}^{\infty} \frac{(a ; q)_{n}(}{\left(a q^{2} ; q^{2}\right)_{n}(b ; q)_{n}(c ; q)_{n}(d ; q)_{n}(e ; q)_{n}(f ; q)_{n}} \\
\quad \times\left(\frac{a^{2} q^{2}}{b c d e f}\right)^{n} \\
=\frac{(a q ; q)_{\infty}(a q / d e ; q)_{\infty}(a q / d f ; q)_{\infty}(a q / e f ; q)_{\infty}}{(a q / d ; q)_{\infty}(a q / e ; q)_{\infty}(a q / f ; q)_{\infty}(a q / d e f ; q)_{\infty}} \\
\quad \times \sum_{n=0}^{\infty} \frac{(a q / b c ; q)_{n}(d ; q)_{n}(e ; q)_{n}(f ; q)_{n}}{(q ; q)_{n}(a q / b ; q)_{n}(a q / c ; q)_{n}(d e f / a ; q)_{n}} q^{n}
\end{aligned}
$$

The proof of Theorem 5 is inspired by that of Theorem 3 . 
TheOREM 5. For a complex number $q$ with $|q|<1$, and $x$ neither 0 nor an integral power of $q$,

$$
f\left(q^{1 / 2}, q^{3 / 2}\right) h(x, q)=\sum_{n=-\infty}^{\infty} \frac{q^{n(n+1 / 2)}}{1-x q^{n}} .
$$

Proof. If we replace $a, b, c$, and $d$ by $1, x, x^{-1}$, and $q^{1 / 2}$, respectively, in (12), let $e, f \rightarrow \infty$ in (12), and use $\lim _{\alpha \rightarrow \infty}(\alpha ; q)_{n} / \alpha^{n}=(-1)^{n} q^{n(n-1) / 2}$ for any positive integer $n$, then we find that (12) becomes

$$
\begin{aligned}
& 1+\sum_{n=1}^{\infty} \frac{\left(1+q^{n}\right)(1-x)\left(1-x^{-1}\right)}{\left(1-x q^{n}\right)\left(1-x^{-1} q^{n}\right)} q^{n^{2}+n / 2} \\
& \quad=\frac{(q ; q)_{\infty}}{\left(q^{1 / 2} ; q\right)_{\infty}} \sum_{n=0}^{\infty} \frac{\left(q^{1 / 2} ; q\right)_{n}(-1)^{n} q^{n^{2} / 2}}{(x q ; q)_{n}\left(x^{-1} q ; q\right)_{n}} .
\end{aligned}
$$

We now consider the left hand side of (14). By a straightforward calculation, we find that the left hand side of (14) equals

$$
\begin{aligned}
& \text { (15) } 1+\sum_{n=1}^{\infty}\left(1+q^{n}\right) q^{n^{2}-n / 2}\left(1-\frac{1-q^{n}}{\left(1+q^{n}\right)\left(1-x q^{n}\right)}-\frac{\left(1-q^{n}\right) x^{-1} q^{n}}{\left(1+q^{n}\right)\left(1-x^{-1} q^{n}\right)}\right) \\
& =1+\sum_{n=1}^{\infty}\left(1+q^{n}\right) q^{n^{2}-n / 2}-\sum_{n=1}^{\infty} \frac{1-q^{n}}{1-x q^{n}} q^{n^{2}-n / 2}-\sum_{n=1}^{\infty} \frac{\left(1-q^{n}\right) x^{-1} q^{n}}{1-x^{-1} q^{n}} q^{n^{2}-n / 2} .
\end{aligned}
$$

By the definition of $f(b, c)$,

$$
1+\sum_{n=1}^{\infty}\left(1+q^{n}\right) q^{n^{2}-n / 2}=\sum_{n=-\infty}^{\infty} q^{n^{2}+n / 2}=f\left(q^{1 / 2}, q^{3 / 2}\right) .
$$

Replacing $n$ by $-n$ in the second sum of the right hand side of (15), and using the equation

$$
\frac{1-q^{-n}}{1-x q^{-n}} \frac{x^{-1} q^{n}}{x^{-1} q^{n}}=\frac{1-q^{n}}{1-x^{-1} q^{n}} x^{-1}
$$

we find that

$$
\sum_{n=-1}^{-\infty} \frac{1-q^{-n}}{1-x q^{-n}} q^{n^{2}+n / 2}=\sum_{n=-1}^{-\infty} \frac{1-q^{n}}{1-x^{-1} q^{n}} x^{-1} q^{n^{2}+n / 2} .
$$

By (16) and (17), the right hand side of (15) is equal to

$$
f\left(q^{1 / 2}, q^{3 / 2}\right)-\sum^{\prime} \frac{\left(1-q^{n}\right) x^{-1}}{1-x^{-1} q^{n}} q^{n^{2}+n / 2},
$$

where $\sum^{\prime}$ means $\sum_{n=-\infty, n \neq 0}^{\infty}$. Thus, multiplying both sides of (14) by $\left(q^{1 / 2} ; q\right)_{\infty} /(q ; q)_{\infty}$, combining the results (14), (15), and (18), replacing $x$ 
by $x^{-1}$, and using (10), we deduce that

$$
\sum_{n=0}^{\infty} \frac{\left(q^{1 / 2} ; q\right)_{n}(-1)^{n} q^{n^{2} / 2}}{(x q ; q)_{n}\left(x^{-1} q ; q\right)_{n}}=1-\frac{x}{f\left(q^{1 / 2}, q^{3 / 2}\right)} \sum^{\prime} \frac{1-q^{n}}{1-x q^{n}} q^{n^{2}+n / 2} .
$$

We easily verify that

$$
\begin{aligned}
& \sum^{\prime} \frac{1-q^{n}}{1-x q^{n}} q^{n^{2}+n / 2} \\
& =\sum^{\prime} \frac{q^{n^{2}+n / 2}}{1-x q^{n}}-\sum^{\prime} q^{n^{2}+n / 2}-1+\sum_{n=-\infty}^{\infty} q^{n^{2}+n / 2}-\sum^{\prime} \frac{q^{n^{2}+3 n / 2}}{1-x q^{n}} \\
& =\sum^{\prime} \frac{q^{n^{2}+n / 2}}{1-x q^{n}}\left(1-\left(1-x q^{n}\right)\right)-1+f\left(q^{1 / 2}, q^{3 / 2}\right)-\sum^{\prime} \frac{q^{n^{2}+3 n / 2}}{1-x q^{n}} \\
& =-(1-x) \sum^{\prime} \frac{q^{n^{2}+3 n / 2}}{1-x q^{n}}-1+f\left(q^{1 / 2}, q^{3 / 2}\right) \\
& =-(1-x) \sum_{n=-\infty}^{\infty} \frac{q^{n^{2}+3 n / 2}}{1-x q^{n}}+f\left(q^{1 / 2}, q^{3 / 2}\right)
\end{aligned}
$$

and

$$
\begin{aligned}
\sum_{n=-\infty}^{\infty} \frac{q^{n^{2}+3 n / 2}}{1-x q^{n}} & =\sum_{n=-\infty}^{\infty} \frac{q^{n^{2}+3 n / 2}-x^{-1} q^{n^{2}+n / 2}+x^{-1} q^{n^{2}+n / 2}}{1-x q^{n}} \\
& =-x^{-1} \sum_{n=-\infty}^{\infty} q^{n^{2}+n / 2}+x^{-1} \sum_{n=-\infty}^{\infty} \frac{q^{n^{2}+n / 2}}{1-x q^{n}} \\
& =-x^{-1} f\left(q^{1 / 2}, q^{3 / 2}\right)+x^{-1} \sum_{n=-\infty}^{\infty} \frac{q^{n^{2}+n / 2}}{1-x q^{n}}
\end{aligned}
$$

Using (19)-(21), we find that

$$
\begin{aligned}
& \sum_{n=0}^{\infty} \frac{\left(q^{1 / 2} ; q\right)_{n}(-1)^{n} q^{n^{2} / 2}}{(x q ; q)_{n}\left(x^{-1} q ; q\right)_{n}} \\
& \quad=1-\frac{x}{f\left(q^{1 / 2}, q^{3 / 2}\right)}\left(-(1-x) \sum_{n=-\infty}^{\infty} \frac{q^{n^{2}+3 n / 2}}{1-x q^{n}}+f\left(q^{1 / 2}, q^{3 / 2}\right)\right) \\
& \quad=1-x+\frac{x(1-x)}{f\left(q^{1 / 2}, q^{3 / 2}\right)}\left(-x^{-1} f\left(q^{1 / 2}, q^{3 / 2}\right)+x^{-1} \sum_{n=-\infty}^{\infty} \frac{q^{n^{2}+n / 2}}{1-x q^{n}}\right) \\
& \quad=\frac{1-x}{f\left(q^{1 / 2}, q^{3 / 2}\right)} \sum_{n=-\infty}^{\infty} \frac{q^{n^{2}+n / 2}}{1-x q^{n}} .
\end{aligned}
$$


Dividing both sides of $(22)$ by $(1-x) / f\left(q^{1 / 2}, q^{3 / 2}\right)$, using the equation

$$
(1-x)(x q ; q)_{n}=(x ; q)_{n+1}
$$

for a nonnegative integer $n$, and using the definition of $h(x, q)$, we verify that

$$
\begin{aligned}
f\left(q^{1 / 2}, q^{3 / 2}\right) & h(x, q) \\
= & f\left(q^{1 / 2}, q^{3 / 2}\right) \sum_{n=0}^{\infty} \frac{\left(q^{1 / 2} ; q\right)_{n}(-1)^{n} q^{n^{2} / 2}}{(x q ; q)_{n+1}\left(x^{-1} q ; q\right)_{n}}=\sum_{n=-\infty}^{\infty} \frac{q^{n^{2}+n / 2}}{1-x q^{n}} .
\end{aligned}
$$

To prove Lemmas 1 and 2, we need Ramanujan's ${ }_{1} \Psi_{1}$ summation.

Theorem 6 (Ramanujan's ${ }_{1} \Psi_{1}$ summation, [2, (17.6), p. 34]). For complex numbers $q, a, b$, and $z$ with $|q|<1$ and $|b / a|<|z|<1$,

$$
\sum_{n=-\infty}^{\infty} \frac{(a ; q)_{n}}{(b ; q)_{n}} z^{n}=\frac{(q ; q)_{\infty}(b / a ; q)_{\infty}(a z ; q)_{\infty}(q / a z ; q)_{\infty}}{(b ; q)_{\infty}(q / a ; q)_{\infty}(z ; q)_{\infty}(b / a z ; q)_{\infty}}
$$

LEMMA 1. For a complex number $q$ with $|q|<1$, and $x$ neither 0 nor an integral power of $q$,

$$
\sum_{n=-\infty}^{\infty} \frac{(-1)^{n} q^{\left(n^{2}+3 n\right) / 2}}{1-x q^{n}}=x^{-1} \frac{(q ; q)_{\infty}^{2}}{(x ; q)_{\infty}\left(x^{-1} q ; q\right)_{\infty}}
$$

Proof. It is easy to verify that

$$
\begin{aligned}
& \sum_{n=-\infty}^{\infty} \frac{(-1)^{n} q^{\left(n^{2}+3 n\right) / 2}}{1-x q^{n}} \\
& \quad=\text { the coefficient of } z^{0} \text { in } \sum_{n=-\infty}^{\infty} \frac{z^{n}}{1-x q^{n}} \sum_{n=-\infty}^{\infty}(-1)^{n} q^{\left(n^{2}+3 n\right) / 2} z^{-n} .
\end{aligned}
$$

Applying Ramanujan's ${ }_{1} \Psi_{1}$ summation with $a$ and $b$ replaced by $x$ and $x q$, respectively, dividing both sides of (23) by $1-x$, applying the Jacobi triple product identity with $b$ and $c$ replaced by $-z q^{-1}$ and $-z^{-1} q^{2}$, respectively, and using $f\left(-z q^{-1},-z^{-1} q^{2}\right)=\left(-z q^{-1}\right) f\left(-z,-z^{-1} q\right)$, we find that the right hand side of (25) equals

$$
\begin{aligned}
& \text { coeff. of } z^{0} \text { in } \frac{(q ; q)_{\infty}^{2} f\left(-x z,-x^{-1} z^{-1} q\right)}{(x ; q)_{\infty}\left(x^{-1} q ; q\right)_{\infty} f\left(-z,-z^{-1} q\right)} f\left(-z q^{-1},-z^{-1} q^{2}\right) \\
& =-q^{-1} \frac{(q ; q)_{\infty}^{2}}{(x ; q)_{\infty}\left(x^{-1} q ; q\right)_{\infty}} \times\left(\text { coeff. of } z^{-1} \text { in } f\left(-x z,-x^{-1} z^{-1} q\right)\right)
\end{aligned}
$$

Therefore, since $f\left(-x z,-x^{-1} z^{-1} q\right)=\sum_{n=-\infty}^{\infty}(-1)^{n} q^{\left(n^{2}-n\right) / 2} x^{n} z^{n}$ by the definition of $f(b, c)$, and the coefficient of $z^{-1}$ in $\sum_{n=-\infty}^{\infty}(-1)^{n} q^{\left(n^{2}-n\right) / 2} x^{n} z^{n}$ 
is $-x^{-1} q$, we find that

$$
\begin{aligned}
\sum_{n=-\infty}^{\infty} \frac{(-1)^{n} q^{\left(n^{2}+3 n\right) / 2}}{1-x q^{n}} & =-q^{-1} \frac{(q ; q)_{\infty}^{2}}{(x ; q)_{\infty}\left(x^{-1} q ; q\right)_{\infty}}\left(-x^{-1} q\right) \\
& =x^{-1} \frac{(q ; q)_{\infty}^{2}}{(x ; q)_{\infty}\left(x^{-1} q ; q\right)_{\infty}} .
\end{aligned}
$$

Lemma 2. For a complex number $q$ with $|q|<1$, and $x$ neither 0 nor an integral power of $q$,

$$
\sum_{n=-\infty}^{\infty} \frac{(-1)^{n} q^{n^{2}+n}}{1-x q^{2 n}}=\frac{\left(q^{2} ; q^{2}\right)_{\infty}^{2}}{\left(x ; q^{2}\right)_{\infty}\left(x^{-1} q^{2} ; q^{2}\right)_{\infty}} .
$$

Proof. It is easy to verify that

$$
\begin{aligned}
& \sum_{n=-\infty}^{\infty} \frac{(-1)^{n} q^{n^{2}+n}}{1-x q^{2 n}} \\
& \quad=\text { the coefficient of } z^{0} \text { in } \sum_{n=-\infty}^{\infty} \frac{z^{n}}{1-x q^{2 n}} \sum_{n=-\infty}^{\infty}(-1)^{n} q^{n^{2}+n} z^{-n}
\end{aligned}
$$

Applying Ramanujan's ${ }_{1} \Psi_{1}$ summation with $q, a$, and $b$ replaced by $q^{2}, x$, and $x q^{2}$, respectively, dividing both sides of (23) by $1-x$, and applying the Jacobi triple product identity with $b$ and $c$ replaced by $-z$ and $-z^{-1} q^{2}$, respectively, we find that the right hand side of (28) equals

$$
\begin{aligned}
& \text { coeff. of } z^{0} \text { in } \frac{\left(q^{2} ; q^{2}\right)_{\infty}^{2} f\left(-x z,-x^{-1} z^{-1} q^{2}\right)}{\left(x ; q^{2}\right)_{\infty}\left(x^{-1} q^{2} ; q^{2}\right)_{\infty} f\left(-z,-z^{-1} q^{2}\right)} f\left(-z,-z^{-1} q^{2}\right) \\
& =\frac{\left(q^{2} ; q^{2}\right)_{\infty}^{2}}{\left(x ; q^{2}\right)_{\infty}\left(x^{-1} q^{2} ; q^{2}\right)_{\infty}} \times\left(\operatorname{coeff} \text {. of } z^{0} \text { in } f\left(-x z,-x^{-1} z^{-1} q^{2}\right)\right) .
\end{aligned}
$$

Therefore, since $f\left(-x z,-x^{-1} z^{-1} q^{2}\right)$ equals $\sum_{n=-\infty}^{\infty}(-1)^{n} q^{n^{2}-n} x^{n} z^{n}$ by the definition of $f(b, c)$, and the coefficient of $z^{0}$ in $\sum_{n=-\infty}^{\infty}(-1)^{n} q^{n^{2}-n} x^{n} z^{n}$ is 1 , we deduce that

$$
\sum_{n=-\infty}^{\infty} \frac{(-1)^{n} q^{n^{2}+n}}{1-x q^{2 n}}=\frac{\left(q^{2} ; q^{2}\right)_{\infty}^{2}}{\left(x ; q^{2}\right)_{\infty}\left(x^{-1} q^{2} ; q^{2}\right)_{\infty}} .
$$

Note that Lemmas 1 and 2 are special cases of the following equation which was obtained by R. J. Evans [7, eq. (3.1)]: for all $x \neq 0$,

$$
\frac{(q ; q)_{\infty}^{2}}{(x q ; q)_{\infty}\left(x^{-1} q ; q\right)_{\infty}}=\sum_{n=-\infty}^{\infty} \frac{(-1)^{n} q^{\left(n^{2}+n\right) / 2}(x-1)}{x-q^{n}} .
$$

We are now ready to prove the main theorems of this paper.

Proof of Theorem 1. Separating the left hand side of (24) according to the residue classes $n \equiv 0(\bmod 3), n \equiv 1(\bmod 3)$, and $n \equiv 2(\bmod 3)$, and 
dividing both sides of (24) by $x^{-1}$, we find that

$$
\begin{aligned}
& \frac{(q ; q)_{\infty}^{2}}{(x ; q)_{\infty}\left(x^{-1} q ; q\right)_{\infty}} \\
& =x \sum_{n=-\infty}^{\infty} \frac{(-1)^{n} q^{\left(9 n^{2}+9 n\right) / 2}}{1-x q^{3 n}}-x \sum_{n=-\infty}^{\infty} \frac{(-1)^{n} q^{\left(9 n^{2}+15 n+4\right) / 2}}{1-x q^{3 n+1}} \\
& \quad+x \sum_{n=-\infty}^{\infty} \frac{(-1)^{n} q^{\left(9 n^{2}+21 n+10\right) / 2}}{1-x q^{3 n+2}}
\end{aligned}
$$

Here, we need to modify the three sums of the right hand side of (30), separately.

For the first sum of the right hand side of (30), applying Theorem 3 with $q$ replaced by $q^{3}$, multiplying both sides of (11) by $x$, and applying the definition of $g(x, q)$ with $q$ replaced by $q^{3}$, we find that

$$
\begin{aligned}
x \sum_{n=-\infty}^{\infty} \frac{(-1)^{n} q^{\left(9 n^{2}+9 n\right) / 2}}{1-x q^{3 n}} & =x\left(q^{3} ; q^{3}\right)_{\infty} g\left(x, q^{3}\right) \\
& =\left(q^{3} ; q^{3}\right)_{\infty}\left(\sum_{n=0}^{\infty} \frac{q^{3 n^{2}}}{\left(x ; q^{3}\right)_{n+1}\left(x^{-1} q^{3} ; q^{3}\right)_{n}}-1\right) .
\end{aligned}
$$

For the second sum of the right hand side of (30), we need the identity

$$
\begin{aligned}
& x \sum_{n=-\infty}^{\infty} \frac{(-1)^{n} q^{\left(9 n^{2}+15 n+4\right) / 2}}{1-x q^{3 n+1}} \\
= & \sum_{n=-\infty}^{\infty} \frac{(-1)^{n} q^{\left(9 n^{2}+9 n\right) / 2+1}\left(x q^{3 n+1}-1\right)}{1-x q^{3 n+1}}+\sum_{n=-\infty}^{\infty} \frac{(-1)^{n} q^{\left(9 n^{2}+9 n\right) / 2+1}}{1-x q^{3 n+1}} \\
= & -\sum_{n=-\infty}^{\infty}(-1)^{n} q^{\left(9 n^{2}+9 n\right) / 2+1}+\sum_{n=-\infty}^{\infty} \frac{(-1)^{n} q^{\left(9 n^{2}+9 n\right) / 2+1}}{1-x q^{3 n+1}} .
\end{aligned}
$$

Using the Jacobi triple product identity with $b$ and $c$ replaced by $-q^{9}$ and -1 , respectively, and using the fact that $\left(1 ; q^{9}\right)_{\infty}=0$, we find that the first sum of the right hand side of (32) equals

$$
q f\left(-q^{9},-1\right)=0 \text {. }
$$

Moreover, applying Theorem 3 with $q$ and $x$ replaced by $q^{3}$ and $x q$, respectively, multiplying both sides of (11) by $q$, and applying the definition of $g(x, q)$ with $q$ and $x$ replaced by $q^{3}$ and $x q$, respectively, we find that the second sum of the right hand side of (32) equals

$$
x^{-1}\left(q^{3} ; q^{3}\right)_{\infty}\left(\sum_{n=0}^{\infty} \frac{q^{3 n^{2}}}{\left(x q ; q^{3}\right)_{n+1}\left(x^{-1} q^{2} ; q^{3}\right)_{n}}-1\right) .
$$


Thus, by (32)-(34), we find that the second sum of the right hand side of (30) becomes (34).

For the third sum of the right hand side of (30), we need the following identity:

$$
\begin{aligned}
& x \sum_{n=-\infty}^{\infty} \frac{(-1)^{n} q^{\left(9 n^{2}+21 n+10\right) / 2}}{1-x q^{3 n+2}} \\
= & \sum_{n=-\infty}^{\infty} \frac{(-1)^{n} q^{\left(9 n^{2}+15 n\right) / 2+3}\left(x q^{3 n+2}-1\right)}{1-x q^{3 n+2}}+\sum_{n=-\infty}^{\infty} \frac{(-1)^{n} q^{\left(9 n^{2}+15 n\right) / 2+3}}{1-x q^{3 n+2}} \\
= & -\sum_{n=-\infty}^{\infty}(-1)^{n} q^{\left(9 n^{2}+15 n\right) / 2+3}+\sum_{n=-\infty}^{\infty} \frac{(-1)^{n} q^{\left(9 n^{2}+15 n\right) / 2+3}}{1-x q^{3 n+2}}
\end{aligned}
$$

Replacing $n$ by $-n-1$ in the first sum of the right hand side of (35), using the Jacobi triple product identity with $b$ and $c$ replaced by $-q^{6}$ and $-q^{3}$, respectively, and using the definition of $f(b, c)$, we find that the first sum of the right hand side of (35) equals

$$
\sum_{n=-\infty}^{\infty}(-1)^{n+1} q^{\left(9 n^{2}+3 n\right) / 2}=-f\left(-q^{6},-q^{3}\right)=-\left(q^{3} ; q^{3}\right)_{\infty} .
$$

Moreover, replacing $n$ by $-n-1$ in the second sum of the right hand side of (35), using

$$
\frac{1}{1-x q^{-3 n-1}}=-\frac{x^{-1} q^{3 n+1}}{1-x^{-1} q^{3 n+1}},
$$

and applying Theorem 3 with $q$ and $x$ replaced by $q^{3}$ and $x^{-1} q$, respectively, and the definition of $g(x, q)$ with $q$ and $x$ replaced by $q^{3}$ and $x^{-1} q$, respectively, we find that the second sum of the right hand side of (35) equals

$$
\begin{aligned}
\sum_{n=-\infty}^{\infty} \frac{(-1)^{n+1} q^{\left(9 n^{2}+3 n\right) / 2}}{1-x q^{-3 n-1}} & =\sum_{n=-\infty}^{\infty} \frac{(-1)^{n} x^{-1} q^{\left(9 n^{2}+9 n\right) / 2+1}}{1-x^{-1} q^{3 n+1}} \\
=x^{-1} q\left(q^{3} ; q^{3}\right)_{\infty} g\left(x^{-1} q, q^{3}\right) & \\
= & \left(q^{3} ; q^{3}\right)_{\infty}\left(\sum_{n=0}^{\infty} \frac{q^{3 n^{2}}}{\left(x^{-1} q ; q^{3}\right)_{n+1}\left(x q^{2} ; q^{3}\right)_{n}}-1\right)
\end{aligned}
$$

Therefore, by (35)-(37), the third sum of the right hand side of (30) becomes

$$
\left(q^{3} ; q^{3}\right)_{\infty}+\left(q^{3} ; q^{3}\right)_{\infty}\left(\sum_{n=0}^{\infty} \frac{q^{3 n^{2}}}{\left(x^{-1} q ; q^{3}\right)_{n+1}\left(x q^{2} ; q^{3}\right)_{n}}-1\right) .
$$


Dividing both sides of $(30)$ by $\left(q^{3} ; q^{3}\right)_{\infty}$, and combining the previous results (30), (31), (34), and (38), we find that

$$
\begin{aligned}
& \frac{(q ; q)_{\infty}^{2}}{(x ; q)_{\infty}\left(x^{-1} q ; q\right)_{\infty}\left(q^{3} ; q^{3}\right)_{\infty}} \\
& =\sum_{n=0}^{\infty} \frac{q^{3 n^{2}}}{\left(x ; q^{3}\right)_{n+1}\left(x^{-1} q^{3} ; q^{3}\right)_{n}}-1-x^{-1}\left(\sum_{n=0}^{\infty} \frac{q^{3 n^{2}}}{\left(x q ; q^{3}\right)_{n+1}\left(x^{-1} q^{2} ; q^{3}\right)_{n}}-1\right) \\
& \quad+\sum_{n=0}^{\infty} \frac{q^{3 n^{2}}}{\left(x^{-1} q ; q^{3}\right)_{n+1}\left(x q^{2} ; q^{3}\right)_{n}} . \text { - }
\end{aligned}
$$

Proof of Theorem 2. Separating the left hand side of (27) according to the residue classes $n \equiv 0(\bmod 2)$ and $n \equiv 1(\bmod 2)$, and dividing both sides of (27) by $x^{-1}$, we find that

$$
\frac{\left(q^{2} ; q^{2}\right)_{\infty}^{2}}{\left(x ; q^{2}\right)_{\infty}\left(x^{-1} q^{2} ; q^{2}\right)_{\infty}}=\sum_{n=-\infty}^{\infty} \frac{q^{4 n^{2}+2 n}}{1-x q^{4 n}}-\sum_{n=-\infty}^{\infty} \frac{q^{4 n^{2}+6 n+2}}{1-x q^{4 n+2}} .
$$

Here, we need to modify the two sums of the right hand side of (39), separately.

For the first sum of the right hand side of (39), applying Theorem 5 with $q$ replaced by $q^{4}$, multiplying both sides of (13) by $x$, and applying the definition of $h(x, q)$ with $q$ replaced by $q^{4}$, we find that

$$
\begin{aligned}
\sum_{n=-\infty}^{\infty} \frac{q^{4 n^{2}+2 n}}{1-x q^{4 n}} & =f\left(q^{2}, q^{6}\right) h\left(x, q^{4}\right) \\
& =f\left(q^{2}, q^{6}\right) \sum_{n=0}^{\infty} \frac{(-1)^{n}\left(q^{2} ; q^{4}\right)_{n} q^{2 n^{2}}}{\left(x ; q^{4}\right)_{n+1}\left(x^{-1} q^{4} ; q^{4}\right)_{n}} .
\end{aligned}
$$

For the second sum of the right hand side of (39), we need the identity

$$
\begin{aligned}
\sum_{n=-\infty}^{\infty} \frac{q^{4 n^{2}+6 n+2}}{1-x} q^{4 n+2} & \\
= & \sum_{n=-\infty}^{\infty} \frac{q^{4 n^{2}+2 n} x^{-1}\left(x q^{4 n+2}-1\right)}{1-x q^{4 n+2}}+\sum_{n=-\infty}^{\infty} \frac{q^{4 n^{2}+2 n} x^{-1}}{1-x q^{4 n+2}} \\
& =-x^{-1} \sum_{n=-\infty}^{\infty} q^{4 n^{2}+2 n}+x^{-1} \sum_{n=-\infty}^{\infty} \frac{q^{4 n^{2}+2 n}}{1-x q^{4 n+2}} .
\end{aligned}
$$

Using the Jacobi triple product identity with $b$ and $c$ replaced by $q^{6}$ and $q^{2}$, respectively, we find that the first sum of the right hand side of (41) equals

$$
x^{-1} f\left(q^{6}, q^{2}\right) \text {. }
$$


Moreover, applying Theorem 5 with $q$ and $x$ replaced by $q^{4}$ and $x q^{2}$, respectively, multiplying both sides of (13) by $x^{-1}$, and applying the definition of $h(x, q)$ with $q$ and $x$ replaced by $q^{4}$ and $x q^{2}$, respectively, we find that the second sum of the right hand side of (41) equals

$$
x^{-1} f\left(q^{2}, q^{6}\right) \sum_{n=0}^{\infty} \frac{(-1)^{n}\left(q^{2} ; q^{4}\right)_{n} q^{2 n^{2}}}{\left(x q^{2} ; q^{4}\right)_{n+1}\left(x^{-1} q^{2} ; q^{4}\right)_{n}} .
$$

Thus, by (41)-(43), we find that the second sum of the right hand side of (39) becomes

$$
-x^{-1} f\left(q^{2}, q^{6}\right)+x^{-1} f\left(q^{2}, q^{6}\right) \sum_{n=0}^{\infty} \frac{(-1)^{n}\left(q^{2} ; q^{4}\right)_{n} q^{2 n^{2}}}{\left(x q^{2} ; q^{4}\right)_{n+1}\left(x^{-1} q^{2} ; q^{4}\right)_{n}} .
$$

Dividing both sides of (39) by $f\left(q^{2}, q^{6}\right)$, and combining the previous results (39), (40) and (44), we find that

$$
\begin{aligned}
& \frac{\left(q^{2} ; q^{2}\right)_{\infty}^{2}}{f\left(q^{2}, q^{6}\right)\left(x ; q^{2}\right)_{\infty}\left(x^{-1} q^{2} ; q^{2}\right)_{\infty}} \\
& \quad=\sum_{n=0}^{\infty} \frac{(-1)^{n}\left(q^{2} ; q^{4}\right)_{n} q^{2 n^{2}}}{\left(x ; q^{4}\right)_{n+1}\left(x^{-1} q^{4} ; q^{4}\right)_{n}}-x^{-1}\left(\sum_{n=0}^{\infty} \frac{(-1)^{n}\left(q^{2} ; q^{4}\right)_{n} q^{2 n^{2}}}{\left(x q^{2} ; q^{4}\right)_{n+1}\left(x^{-1} q^{2} ; q^{4}\right)_{n}}-1\right) .
\end{aligned}
$$

3. Two identities in Ramanujan's lost notebook. In this section, we provide the proofs for two identities in Ramanujan's lost notebook.

TheOREM 7. For any complex number $q$ with $|q|<1$, let

$$
\Psi(q)=\sum_{n=0}^{\infty} \frac{q^{5 n^{2}}}{\left(q^{2} ; q^{5}\right)_{n+1}\left(q^{3} ; q^{5}\right)_{n}}, \quad \Phi(q)=\sum_{n=0}^{\infty} \frac{q^{5 n^{2}}}{\left(q ; q^{5}\right)_{n+1}\left(q^{4} ; q^{5}\right)_{n}} .
$$

Then

$$
\begin{aligned}
& \frac{1}{q}\left(\Psi\left(q^{3}\right)-1\right)+\frac{\left(q^{5} ; q^{5}\right)_{\infty}^{2}}{\left(q^{15} ; q^{15}\right)_{\infty}\left(q ; q^{5}\right)_{\infty}\left(q^{4} ; q^{5}\right)_{\infty}} \\
& \quad=\sum_{n=0}^{\infty} \frac{q^{15 n^{2}}}{\left(q ; q^{15}\right)_{n+1}\left(q^{14} ; q^{15}\right)_{n}}-1+\sum_{n=0}^{\infty} \frac{q^{15 n^{2}}}{\left(q^{4} ; q^{15}\right)_{n+1}\left(q^{11} ; q^{15}\right)_{n}} \\
& \Phi\left(q^{3}\right)-1+\sum_{n=0}^{\infty} \frac{q^{15 n^{2}}}{\left(q^{2} ; q^{15}\right)_{n+1}\left(q^{13} ; q^{15}\right)_{n}} \\
& -\frac{1}{q^{2}}\left(\sum_{n=0}^{\infty} \frac{q^{15 n^{2}}}{\left(q^{7} ; q^{15}\right)_{n+1}\left(q^{8} ; q^{15}\right)_{n}}-1\right)=\frac{\left(q^{5} ; q^{5}\right)_{\infty}^{2}}{\left(q^{15} ; q^{15}\right)_{\infty}\left(q^{2} ; q^{5}\right)_{\infty}\left(q^{3} ; q^{5}\right)_{\infty}}
\end{aligned}
$$


Proof. Applying Theorem 1 with $q$ and $x$ replaced by $q^{5}$ and $q$, respectively, we find that

$$
\begin{aligned}
& \frac{\left(q^{5} ; q^{5}\right)_{\infty}^{2}}{\left(q^{15} ; q^{15}\right)_{\infty}\left(q ; q^{5}\right)_{\infty}\left(q^{4} ; q^{5}\right)_{\infty}}=\sum_{n=0}^{\infty} \frac{q^{15 n^{2}}}{\left(q ; q^{15}\right)_{n+1}\left(q^{14} ; q^{15}\right)_{n}}-1 \\
& -q^{-1}\left(\sum_{n=0}^{\infty} \frac{q^{15 n^{2}}}{\left(q^{6} ; q^{15}\right)_{n+1}\left(q^{9} ; q^{15}\right)_{n}}-1\right)+\sum_{n=0}^{\infty} \frac{q^{15 n^{2}}}{\left(q^{4} ; q^{15}\right)_{n+1}\left(q^{11} ; q^{15}\right)_{n}}
\end{aligned}
$$

Now applying the definition of $\Psi(q)$, and rearranging sums in (47), we derive (45).

Similarly, applying Theorem 1 with $q$ and $x$ replaced by $q^{5}$ and $q^{2}$, respectively, we find that

$$
\begin{aligned}
& \frac{\left(q^{5} ; q^{5}\right)_{\infty}^{2}}{\left(q^{15} ; q^{15}\right)_{\infty}\left(q^{2} ; q^{5}\right)_{\infty}\left(q^{3} ; q^{5}\right)_{\infty}}=\sum_{n=0}^{\infty} \frac{q^{15 n^{2}}}{\left(q^{2} ; q^{15}\right)_{n+1}\left(q^{13} ; q^{15}\right)_{n}}-1 \\
& -q^{-2}\left(\sum_{n=0}^{\infty} \frac{q^{15 n^{2}}}{\left(q^{7} ; q^{15}\right)_{n+1}\left(q^{8} ; q^{15}\right)_{n}}-1\right)+\sum_{n=0}^{\infty} \frac{q^{15 n^{2}}}{\left(q^{3} ; q^{15}\right)_{n+1}\left(q^{12} ; q^{15}\right)_{n}}
\end{aligned}
$$

Now using the definition of $\Phi(q)$, and rearranging sums in (48), we deduce (46).

4. Mock theta functions of third order. In this section, we introduce two new identities for the third order mock theta functions $\psi(q)$ and $\omega(q)$ which are not stated by Ramanujan.

THEOREM 8. For any complex number $q$ with $|q|<1$, let

$$
\psi(q)=\sum_{n=1}^{\infty} \frac{q^{n^{2}}}{\left(q ; q^{2}\right)_{n}}, \quad \omega(q)=\sum_{n=0}^{\infty} \frac{q^{2 n(n+1)}}{\left(q ; q^{2}\right)_{n+1}^{2}} .
$$

Then

$$
\begin{aligned}
& \psi\left(q^{3}\right)+\sum_{n=0}^{\infty} \frac{q^{12 n^{2}}}{\left(q ; q^{12}\right)_{n+1}\left(q^{11} ; q^{12}\right)_{n}}-\frac{1}{q^{3}}\left(\sum_{n=0}^{\infty} \frac{q^{12 n^{2}}}{\left(q^{7} ; q^{12}\right)_{n+1}\left(q^{5} ; q^{12}\right)_{n}}-1\right) \\
& =\frac{\left(q^{4} ; q^{4}\right)_{\infty}^{2}}{\left(q^{12} ; q^{12}\right)_{\infty}\left(q ; q^{4}\right)_{\infty}\left(q^{3} ; q^{4}\right)_{\infty}}, \\
& q^{2} \omega\left(q^{3}\right)+\frac{\left(q^{2} ; q^{2}\right)_{\infty}^{2}}{\left(q^{6} ; q^{6}\right)_{\infty}\left(q ; q^{2}\right)_{\infty}^{2}}=2 \sum_{n=0}^{\infty} \frac{q^{6 n^{2}}}{\left(q ; q^{6}\right)_{n+1}\left(q^{5} ; q^{6}\right)_{n}}-1 .
\end{aligned}
$$

Proof. In [11], Watson proved that Ramanujan's third order mock theta functions $\psi(q)$ and $\omega(q)$ satisfy the identities

$$
\psi(q)=\frac{1}{\left(q^{4} ; q^{4}\right)_{\infty}} \sum_{n=-\infty}^{\infty} \frac{(-1)^{n} q^{6 n(n+1)+1}}{1-q^{4 n+1}},
$$




$$
\omega(q)=\frac{1}{\left(q^{2} ; q^{2}\right)_{\infty}} \sum_{n=-\infty}^{\infty} \frac{(-1)^{n} q^{3 n(n+1)}}{1-q^{2 n+1}} .
$$

Replacing $q$ and $x$ by $q^{4}$ and $q$, respectively, in (11), dividing both sides of (11) by $\left(q^{4} ; q^{4}\right)_{\infty} / q$, and using (51) and the definition of $g(x, q)$ with $q$ and $x$ replaced by $q^{4}$ and $q$, respectively, we find

$$
\psi(q)=\sum_{n=0}^{\infty} \frac{q^{4 n^{2}}}{\left(q ; q^{4}\right)_{n+1}\left(q^{3} ; q^{4}\right)_{n}}-1,
$$

and replacing $q$ and $x$ by $q^{2}$ and $q$, respectively, in (11), dividing both sides of (11) by $\left(q^{2} ; q^{2}\right)_{\infty}$, and using (52) and the definition of $g(x, q)$ with $q$ and $x$ replaced by $q^{2}$ and $q$, respectively, we find

$$
\omega(q)=\frac{1}{q}\left(\sum_{n=0}^{\infty} \frac{q^{2 n^{2}}}{\left(q ; q^{2}\right)_{n+1}\left(q ; q^{2}\right)_{n}}-1\right) .
$$

Applying Theorem 1 with $q$ and $x$ replaced by $q^{4}$ and $q^{3}$, respectively, we have

$$
\begin{aligned}
& \frac{\left(q^{4} ; q^{4}\right)_{\infty}^{2}}{\left(q^{12} ; q^{12}\right)_{\infty}\left(q^{3} ; q^{4}\right)_{\infty}\left(q ; q^{4}\right)_{\infty}}=\sum_{n=0}^{\infty} \frac{q^{12 n^{2}}}{\left(q^{3} ; q^{12}\right)_{n+1}\left(q^{9} ; q^{12}\right)_{n}}-1 \\
& -q^{-3}\left(\sum_{n=0}^{\infty} \frac{q^{12 n^{2}}}{\left(q^{7} ; q^{12}\right)_{n+1}\left(q^{5} ; q^{12}\right)_{n}}-1\right)+\sum_{n=0}^{\infty} \frac{q^{12 n^{2}}}{\left(q ; q^{12}\right)_{n+1}\left(q^{11} ; q^{12}\right)_{n}}
\end{aligned}
$$

Then, using (53) with $q$ replaced by $q^{3}$, and rearranging sums in (55), we complete the proof of (49).

Applying Theorem 1 with $q$ and $x$ replaced by $q^{2}$ and $q$, respectively, we find that

$$
\begin{aligned}
& \frac{\left(q^{2} ; q^{2}\right)_{\infty}^{2}}{\left(q^{6} ; q^{6}\right)_{\infty}\left(q ; q^{2}\right)_{\infty}\left(q ; q^{2}\right)_{\infty}}=\sum_{n=0}^{\infty} \frac{q^{6 n^{2}}}{\left(q ; q^{6}\right)_{n+1}\left(q^{5} ; q^{6}\right)_{n}}-1 \\
& -q^{-1}\left(\sum_{n=0}^{\infty} \frac{q^{6 n^{2}}}{\left(q^{3} ; q^{6}\right)_{n+1}\left(q^{3} ; q^{6}\right)_{n}}-1\right)+\sum_{n=0}^{\infty} \frac{q^{6 n^{2}}}{\left(q ; q^{6}\right)_{n+1}\left(q^{5} ; q^{6}\right)_{n}}
\end{aligned}
$$

Then, using (54) with $q$ replaced by $q^{3}$, and rearranging sums in (56), we complete the proof of $(50)$.

5. Mock theta functions of seventh order. In this section, we introduce three identities related to Ramanujan's seventh order mock theta functions which are not given by Ramanujan. 
TheOREM 9. For any complex number $q$ with $|q|<1$, let

$$
\begin{gathered}
\psi_{0}(q)=\sum_{n=0}^{\infty} \frac{q^{7 n^{2}}}{\left(q ; q^{7}\right)_{n+1}\left(q^{6} ; q^{7}\right)_{n}}, \quad \psi_{1}(q)=\sum_{n=0}^{\infty} \frac{q^{7 n^{2}}}{\left(q^{2} ; q^{7}\right)_{n+1}\left(q^{5} ; q^{7}\right)_{n}}, \\
\psi_{2}(q)=\sum_{n=0}^{\infty} \frac{q^{7 n^{2}}}{\left(q^{3} ; q^{7}\right)_{n+1}\left(q^{4} ; q^{7}\right)_{n}} .
\end{gathered}
$$

Then

$$
\begin{aligned}
& \psi_{0}\left(q^{3}\right)-1+\sum_{n=0}^{\infty} \frac{q^{21 n^{2}}}{\left(q^{4} ; q^{21}\right)_{n+1}\left(q^{17} ; q^{21}\right)_{n}} \\
& -\frac{1}{q^{3}}\left(\sum_{n=0}^{\infty} \frac{q^{21 n^{2}}}{\left(q^{10} ; q^{21}\right)_{n+1}\left(q^{11} ; q^{21}\right)_{n}}-1\right)=\frac{\left(q^{7} ; q^{7}\right)_{\infty}^{2}}{\left(q^{21} ; q^{21}\right)_{\infty}\left(q^{3} ; q^{7}\right)_{\infty}\left(q^{4} ; q^{7}\right)_{\infty}}, \\
& \psi_{1}\left(q^{3}\right)-1+\sum_{n=0}^{\infty} \frac{q^{21 n^{2}}}{\left(q ; q^{21}\right)_{n+1}\left(q^{20} ; q^{21}\right)_{n}} \\
& -\frac{1}{q}\left(\sum_{n=0}^{\infty} \frac{q^{21 n^{2}}}{\left(q^{8} ; q^{21}\right)_{n+1}\left(q^{13} ; q^{21}\right)_{n}}-1\right)=\frac{\left(q^{7} ; q^{7}\right)_{\infty}^{2}}{\left(q^{21} ; q^{21}\right)_{\infty}\left(q ; q^{7}\right)_{\infty}\left(q^{6} ; q^{7}\right)_{\infty}}, \\
& \psi_{2}\left(q^{3}\right)-1+\frac{\left(q^{7} ; q^{7}\right)_{\infty}^{2}}{\left(q^{21} ; q^{21}\right)_{\infty}\left(q^{2} ; q^{7}\right)_{\infty}\left(q^{5} ; q^{7}\right)_{\infty}} \\
& =\sum_{n=0}^{\infty} \frac{q^{21 n^{2}}}{\left(q^{2} ; q^{21}\right)_{n+1}\left(q^{19} ; q^{21}\right)_{n}}-1+\sum_{n=0}^{\infty} \frac{q^{21 n^{2}}}{\left(q^{5} ; q^{21}\right)_{n+1}\left(q^{16} ; q^{21}\right)_{n}}
\end{aligned}
$$

Recall that in [9], Hickerson proved that Ramanujan's seventh order mock theta functions $F_{0}(q), F_{1}(q)$ and $F_{2}(q)$ satisfy the identities

$$
\begin{aligned}
& F_{0}(q)=\frac{2 q}{\left(q^{7} ; q^{7}\right)_{\infty}} \sum_{n=-\infty}^{\infty} \frac{(-1)^{n} q^{21 n(n+1) / 2}}{1-q^{7 n+1}}+2-\frac{f\left(-q^{3},-q^{4}\right)^{2}}{(q ; q)_{\infty}}, \\
& F_{1}(q)=\frac{2 q^{2}}{\left(q^{7} ; q^{7}\right)_{\infty}} \sum_{n=-\infty}^{\infty} \frac{(-1)^{n} q^{21 n(n+1) / 2}}{1-q^{7 n+2}}+\frac{q f\left(-q,-q^{6}\right)^{2}}{(q ; q)_{\infty}} \\
& F_{2}(q)=\frac{2 q^{2}}{\left(q^{7} ; q^{7}\right)_{\infty}} \sum_{n=-\infty}^{\infty} \frac{(-1)^{n} q^{21 n(n+1) / 2}}{1-q^{7 n+3}}+\frac{f\left(-q^{2},-q^{5}\right)^{2}}{(q ; q)_{\infty}} .
\end{aligned}
$$

Applying Theorem 3 with $q$ and $x$ replaced by $q^{7}$ and $q$, respectively, dividing both sides of $(11)$ by $\left(q^{7} ; q^{7}\right)_{\infty}$, using the definition of $g(x, q)$ with $q$ and $x$ replaced by $q^{7}$ and $q$, respectively, and the definition of $\psi_{0}(q)$, we deduce that the identity for $F_{0}(q)$ above is equivalent to

$$
F_{0}(q)=2 \psi_{0}(q)-\frac{f\left(-q^{3},-q^{4}\right)^{2}}{(q ; q)_{\infty}} .
$$


Applying Theorem 3 with $q$ and $x$ replaced by $q^{7}$ and $q^{2}$, respectively, dividing both sides of $(11)$ by $\left(q^{7} ; q^{7}\right)_{\infty}$, using the definition of $g(x, q)$ with $q$ and $x$ replaced by $q^{7}$ and $q^{2}$, respectively, and the definition of $\psi_{1}(q)$, we deduce that the identity for $F_{1}(q)$ above is equivalent to

$$
F_{1}(q)=2 \psi_{1}(q)+\frac{q f\left(-q,-q^{6}\right)^{2}}{(q ; q)_{\infty}} .
$$

Applying Theorem 3 with $q$ and $x$ replaced by $q^{7}$ and $q^{3}$, respectively, dividing both sides of $(11)$ by $\left(q^{7} ; q^{7}\right)_{\infty}$, using the definition of $g(x, q)$ with $q$ and $x$ replaced by $q^{7}$ and $q^{3}$, respectively, and the definition of $\psi_{2}(q)$, we deduce that the identity for $F_{2}(q)$ above is equivalent to

$$
F_{2}(q)=2 q^{-1}\left(\psi_{2}(q)-1\right)+\frac{f\left(-q^{2},-q^{5}\right)^{2}}{(q ; q)_{\infty}} .
$$

Proof of Theorem 9. Applying Theorem 1 with $q$ and $x$ replaced by $q^{7}$ and $q^{3}$, respectively, we find that

$$
\begin{gathered}
\frac{\left(q^{7} ; q^{7}\right)_{\infty}^{2}}{\left(q^{21} ; q^{21}\right)_{\infty}\left(q^{3} ; q^{7}\right)_{\infty}\left(q^{4} ; q^{7}\right)_{\infty}}=\sum_{n=0}^{\infty} \frac{q^{21 n^{2}}}{\left(q^{3} ; q^{21}\right)_{n+1}\left(q^{18} ; q^{21}\right)_{n}}-1 \\
-q^{-3}\left(\sum_{n=0}^{\infty} \frac{q^{21 n^{2}}}{\left(q^{10} ; q^{21}\right)_{n+1}\left(q^{11} ; q^{21}\right)_{n}}-1\right)+\sum_{n=0}^{\infty} \frac{q^{21 n^{2}}}{\left(q^{4} ; q^{21}\right)_{n+1}\left(q^{17} ; q^{21}\right)_{n}}
\end{gathered}
$$

Then, using the definition of $\psi_{0}(q)$ with $q$ replaced by $q^{3}$, and rearranging sums in (60), we find (57).

Applying Theorem 1 with $q$ and $x$ replaced by $q^{7}$ and $q$, respectively, we find that

$$
\begin{aligned}
& \frac{\left(q^{7} ; q^{7}\right)_{\infty}^{2}}{\left(q^{21} ; q^{21}\right)_{\infty}\left(q ; q^{7}\right)_{\infty}\left(q^{6} ; q^{7}\right)_{\infty}}=\sum_{n=0}^{\infty} \frac{q^{21 n^{2}}}{\left(q ; q^{21}\right)_{n+1}\left(q^{20} ; q^{21}\right)_{n}}-1 \\
& -q^{-1}\left(\sum_{n=0}^{\infty} \frac{q^{21 n^{2}}}{\left(q^{8} ; q^{21}\right)_{n+1}\left(q^{13} ; q^{21}\right)_{n}}-1\right)+\sum_{n=0}^{\infty} \frac{q^{21 n^{2}}}{\left(q^{6} ; q^{21}\right)_{n+1}\left(q^{15} ; q^{21}\right)_{n}}
\end{aligned}
$$

Then, using the definition of $\psi_{1}(q)$ with $q$ replaced by $q^{3}$, and rearranging sums in (61), we deduce (58).

Applying Theorem 1 with $q$ and $x$ replaced by $q^{7}$ and $q^{2}$, respectively, we find that

$$
\begin{aligned}
& \frac{\left(q^{7} ; q^{7}\right)_{\infty}^{2}}{\left(q^{21} ; q^{21}\right)_{\infty}\left(q^{2} ; q^{7}\right)_{\infty}\left(q^{5} ; q^{7}\right)_{\infty}}=\sum_{n=0}^{\infty} \frac{q^{21 n^{2}}}{\left(q^{2} ; q^{21}\right)_{n+1}\left(q^{19} ; q^{21}\right)_{n}}-1 \\
& -q^{-2}\left(\sum_{n=0}^{\infty} \frac{q^{21 n^{2}}}{\left(q^{9} ; q^{21}\right)_{n+1}\left(q^{12} ; q^{21}\right)_{n}}-1\right)+\sum_{n=0}^{\infty} \frac{q^{21 n^{2}}}{\left(q^{5} ; q^{21}\right)_{n+1}\left(q^{16} ; q^{21}\right)_{n}}
\end{aligned}
$$


Using the definition of $\psi_{2}(q)$ with $q$ replaced by $q^{3}$, and rearranging sums in (62), we verify (59).

6. Mock theta functions of tenth order. In this section, we introduce two identities related to Ramanujan's tenth order mock theta functions which are not stated by Ramanujan.

TheOREM 10. For any complex number $q$ with $|q|<1$, let

$$
\phi_{0}(q)=\sum_{n=0}^{\infty} \frac{(-1)^{n}\left(q^{5} ; q^{10}\right)_{n} q^{5 n^{2}}}{\left(q^{2} ; q^{10}\right)_{n+1}\left(q^{8} ; q^{10}\right)_{n}}, \quad \phi_{1}(q)=\sum_{n=0}^{\infty} \frac{(-1)^{n}\left(q^{5} ; q^{10}\right)_{n} q^{5 n^{2}}}{\left(q^{4} ; q^{10}\right)_{n+1}\left(q^{6} ; q^{10}\right)_{n}} .
$$

Then

$$
\begin{gathered}
\phi_{0}\left(q^{2}\right)-\frac{1}{q^{4}}\left(\sum_{n=0}^{\infty} \frac{(-1)^{n}\left(q^{10} ; q^{20}\right)_{n} q^{10 n^{2}}}{\left(q^{14} ; q^{20}\right)_{n+1}\left(q^{6} ; q^{20}\right)_{n}}-1\right) \\
=\frac{\left(q^{10} ; q^{10}\right)_{\infty}^{2}}{\left(q^{4} ; q^{10}\right)_{\infty}\left(q^{6} ; q^{10}\right)_{\infty} f\left(q^{10}, q^{30}\right)}, \\
\phi_{1}\left(q^{2}\right)-\frac{1}{q^{8}}\left(\sum_{n=0}^{\infty} \frac{(-1)^{n}\left(q^{10} ; q^{20}\right)_{n} q^{10 n^{2}}}{\left(q^{18} ; q^{20}\right)_{n+1}\left(q^{2} ; q^{20}\right)_{n}}-1\right) \\
=\frac{\left(q^{10} ; q^{10}\right)_{\infty}^{2}}{\left(q^{2} ; q^{10}\right)_{\infty}\left(q^{8} ; q^{10}\right)_{\infty} f\left(q^{10}, q^{30}\right)} .
\end{gathered}
$$

Recall that in [4], the author proved that Ramanujan's tenth order mock theta functions $X(q)$ and $\chi(q)$ satisfy the identities

$$
\begin{aligned}
& X(q)=-\frac{\left(q^{5} ; q^{5}\right)_{\infty}\left(q^{10} ; q^{10}\right)_{\infty} f\left(-q^{2},-q^{3}\right)}{f\left(-q^{2},-q^{8}\right) f\left(-q,-q^{4}\right)}+\frac{2}{f\left(q^{5}, q^{15}\right)} \sum_{n=-\infty}^{\infty} \frac{q^{5 n(2 n+1)}}{1-q^{10 n+2}} \\
& \chi(q)=2+q \frac{\left(q^{5} ; q^{5}\right)_{\infty}\left(q^{10} ; q^{10}\right)_{\infty} f\left(-q,-q^{4}\right)}{f\left(-q^{4},-q^{6}\right) f\left(-q^{2},-q^{3}\right)}-\frac{2}{f\left(q^{5}, q^{15}\right)} \sum_{n=-\infty}^{\infty} \frac{q^{5 n(2 n+1)}}{1-q^{10 n+4}} .
\end{aligned}
$$

Applying Theorem 5 with $q$ and $x$ replaced by $q^{10}$ and $q^{2}$, respectively, dividing both sides of (13) by $f\left(q^{5}, q^{15}\right)$, using the definition of $h(x, q)$ with $q$ and $x$ replaced by $q^{10}$ and $q^{2}$, respectively, and the definition of $\phi_{0}(q)$, we deduce that the identity for $X(q)$ above is equivalent to

$$
X(q)=-\frac{\left(q^{5} ; q^{5}\right)_{\infty}\left(q^{10} ; q^{10}\right)_{\infty} f\left(-q^{2},-q^{3}\right)}{f\left(-q^{2},-q^{8}\right) f\left(-q,-q^{4}\right)}+2 \phi_{0}(q) .
$$

Applying Theorem 5 with $q$ and $x$ replaced by $q^{10}$ and $q^{4}$, respectively, dividing both sides of (13) by $f\left(q^{5}, q^{15}\right)$, using the definition of $h(x, q)$ with $q$ and $x$ replaced by $q^{10}$ and $q^{4}$, respectively, and the definition of $\phi_{1}(q)$, we 
deduce that the identity for $\chi(q)$ above is equivalent to

$$
\chi(q)=2+q \frac{\left(q^{5} ; q^{5}\right)_{\infty}\left(q^{10} ; q^{10}\right)_{\infty} f\left(-q,-q^{4}\right)}{f\left(-q^{4},-q^{6}\right) f\left(-q^{2},-q^{3}\right)}-2 \phi_{1}(q) .
$$

Proof of Theorem 10. Applying Theorem 2 with $q$ and $x$ replaced by $q^{5}$ and $q^{4}$, respectively, we find that

$$
\begin{aligned}
& \frac{\left(q^{10} ; q^{10}\right)_{\infty}^{2}}{f\left(q^{10}, q^{30}\right)\left(q^{4} ; q^{10}\right)_{\infty}\left(q^{6} ; q^{10}\right)_{\infty}}=\sum_{n=0}^{\infty} \frac{(-1)^{n}\left(q^{10} ; q^{20}\right)_{n} q^{10 n^{2}}}{\left(q^{4} ; q^{20}\right)_{n+1}\left(q^{16} ; q^{20}\right)_{n}} \\
&-q^{-4}\left(\sum_{n=0}^{\infty} \frac{(-1)^{n}\left(q^{10} ; q^{20}\right)_{n} q^{10 n^{2}}}{\left(q^{14} ; q^{20}\right)_{n+1}\left(q^{6} ; q^{20}\right)_{n}}-1\right) .
\end{aligned}
$$

Then, using the definition of $\phi_{0}(q)$ with $q$ replaced by $q^{2}$, and rearranging sums in (65), we deduce (63).

Applying Theorem 2 with $q$ and $x$ replaced by $q^{5}$ and $q^{8}$, respectively, we find that

$$
\begin{aligned}
& \frac{\left(q^{10} ; q^{10}\right)_{\infty}^{2}}{f\left(q^{10}, q^{30}\right)\left(q^{8} ; q^{10}\right)_{\infty}\left(q^{2} ; q^{10}\right)_{\infty}}=\sum_{n=0}^{\infty} \frac{(-1)^{n}\left(q^{10} ; q^{20}\right)_{n} q^{10 n^{2}}}{\left(q^{8} ; q^{20}\right)_{n+1}\left(q^{12} ; q^{20}\right)_{n}} \\
&-q^{-8}\left(\sum_{n=0}^{\infty} \frac{(-1)^{n}\left(q^{10} ; q^{20}\right)_{n} q^{10 n^{2}}}{\left(q^{18} ; q^{20}\right)_{n+1}\left(q^{2} ; q^{20}\right)_{n}}-1\right)
\end{aligned}
$$

Then, using the definition of $\phi_{1}(q)$ with $q$ replaced by $q^{2}$, and rearranging sums in (66), we verify (64).

\section{References}

[1] G. E. Andrews and F. G. Garvan, Ramanujan's "lost" notebook VI: the mock theta conjectures, Adv. Math. 73 (1989), 242-255.

[2] B. C. Berndt, Ramanujan's Notebooks, Part III, Springer, New York, 1991.

[3] B. C. Berndt and R. A. Rankin, Ramanujan: Letters and Commentary, Amer. Math. Soc., Providence, RI, 1995, London Math. Soc., London, 1995.

[4] Y.-S. Choi, Tenth order mock theta functions in Ramanujan's lost notebook (II), Adv. Math. 156 (2000), 180-285.

[5] - , Tenth order mock theta functions in Ramanujan's lost notebook (IV), Trans. Amer. Math. Soc. 354 (2002), 705-733.

[6] - Identities for Ramanujan's sixth-order mock theta functions, Quart. J. Math. 53 (2002), 147-159.

[7] R. J. Evans, Generalized Lambert series, in: Analytic Number Theory (Allerton Park, IL, 1995), Vol. 1, B. C. Berndt, H. G. Diamond, and A. J. Hildebrand (eds.), Birkhäuser, Boston, 1996, 357-370.

[8] D. Hickerson, A proof of the mock theta conjectures, Invent. Math. 94 (1988), 639660.

[9] —, On the seventh order mock theta functions, ibid., 661-677. 
[10] S. Ramanujan, The Lost Notebook and Other Unpublished Papers, Narosa Publishing House, New Delhi, 1988.

[11] G. N. Watson, The final problem: An account of the mock theta functions, J. London Math. Soc. 11 (1936), 55-80.

[12] —, The mock theta functions (2), Proc. London Math. Soc. 42 (1936), 274-304.

Department of Mathematics

Korea University

5-1, Anam-dong

Sungbuk-ku

Seoul 136-701, South Korea

E-mail: y-choi2@korea.ac.kr 